\title{
Onicomicosis en pediatría: Actualización y tratamiento
}

\author{
Pediatric onychomycosis: Update and management
}

\author{
Daniela A. Alfaro S. ${ }^{\text {, }}$, Carmen G. González F. ${ }^{\mathrm{b}}$
}

aEscuela de Medicina, Facultad de Medicina, Pontificia Universidad Católica de Chile, Santiago de Chile

bServicio de Dermatología, Clínica Dávila, Santiago de Chile

Recibido: 27 de junio de 2019; Aceptado: 2 de septiembre de 2019

\section{¿Qué se sabe del tema que trata este estudio?}

La onicomicosis es una patología poco frecuente en niños, cuya prevalencia ha ido en aumento. Si bien en adultos existen múltiples guías clínicas, en población pediátrica la literatura es escasa.

\begin{abstract}
¿Qué aporta este estudio a lo ya conocido?
El presente manuscrito realiza una revisión sobre onicomicosis en población pediátrica, abarcando su epidemiología, microbiología, diagnóstico y tratamiento. Además, discute la utilidad de nuevas técnicas diagnósticas y los resultados de nuevos tratamientos, en la población pediátrica.
\end{abstract}

\section{Resumen}

La onicomicosis $(\mathrm{OM})$ es una infección fúngica de las uñas, cuyo principal agente causal es el Tricophytum rubrum. Si bien es una patología infrecuente en niños, se ha observado un aumento en la prevalencia en el último tiempo. Hasta la fecha, existen diversos estudios y guías clínicas de OM en adultos. Sin embargo, la literatura en edad pediátrica es escasa, lo que dificulta el tratamiento en pediatría. En el presente articulo se revisa la literatura actual, los métodos diagnosticos de OM, datos epidemiológicos locales y globales, y se presentan las opciones de tratamiento disponibles considerando su eficacia y perfil de seguridad en población pediátrica.
Palabras clave: onicomicosis; dermatofitos; tiña;

uña; pediatría

\section{Keywords:} onychomycosis; dermatophytes; tinea; nail; pediatrics 


\section{Introducción}

La onicomicosis $(\mathrm{OM})$ es una infección fúngica crónica y recurrente de la uña. Es una patología cuya prevalencia aumenta con la edad, siendo menos frecuente en niños que en adultos ${ }^{1}$. En ambos grupos, la afectación de los pies predomina sobre las manos, siendo el Trichophyton rubrum (T. rubrum) el principal agente causal ${ }^{2-10}$. En el último tiempo, se ha observado un aumento en la prevalencia de $\mathrm{OM}$ en niños ${ }^{9,10}$.

Debido a que el pediatra es el principal encargado de velar por la salud integral del niño y adolescente; es importante que la OM sea considerada como parte del diagnóstico diferencial cuando se observan alteraciones ungueales en el examen físico en niños.

Por lo anterior, el objetivo de este manuscrito es revisar la literatura actual, para conocer los métodos diagnósticos de $\mathrm{OM}$, los datos epidemiológicos nacionales e internacionales y presentar las opciones de tratamiento disponibles, considerando su eficacia y perfil de seguridad en población pediátrica.

\section{Epidemiología}

Se calcula que la prevalencia de la OM en población general es de aproximadamente un 3,2\%, siendo una patología que aumenta con la edad. Se estima que en niños alcanzaría un $0,14 \%$ y en adultos mayores un $10,2 \%{ }^{1}$. Es probable que esto se deba en parte, a que el crecimiento de la uña en niños es más rápido y a que por ser la uña de los niños más pequeña, exista una menor área de exposición a hongos y con ello menor riesgo de eventual contagio ${ }^{2}$.

Gupta et al., realizaron un estudio prospectivo, multicéntrico, en el que se analizaron los datos de 2.500 niños, referidos a dermatología. La prevalencia total de OM fue $0,44 \%$. Al excluir a aquellos niños referidos por OM, la prevalencia de esta patología como hallazgo fue del $0,16 \%{ }^{2}$. Si bien, este es un estudio sesgado, que fue desarrollado en un centro dermatológico, es interesante destacar a este grupo de pacientes, en quienes su motivo de consulta y derivación primaria fue otro. Lo anterior refuerza la necesidad de realizar un cuidadoso examen físico, ya que la OM puede corresponder a un hallazgo incidental. En la misma publicación, se agregaron datos de otros estudios, llevados a cabo en centros dermatológicos y escuelas de distintos países (México, África, Gales, España, India, Sudán, Canadá, EEUU, Finlandia), en un intento por estimar la prevalencia global, llegando a un valor aproximado de $0,3 \%(\mathrm{~N}=27.930)^{2}$.

En un nuevo trabajo liderado por Gupta et al., se realizó una revisión sistemática sobre la prevalencia de $\mathrm{OM}$ en distintos países, entre los cuales se incluyeron:
Turquía, Israel, España, Canadá, Estados Unidos y Gales. El objetivo fue calcular la prevalencia de OM en distintos grupos: niños, adultos mayores y diabéticos, entre otros. En este análisis se incluyeron un total de 37.004 niños, de entre 3 a 18 años. Al calcular en este grupo la prevalencia de OM, confirmada por cultivo, esta fue de $0,14 \%$, siendo hasta la fecha el mayor estudio realizado en niños ${ }^{1}$.

En Islandia, se analizaron los cultivos de todo el país entre 1982 a 2000, observándose un aumento de la incidencia de 1,7 por 100.000 niños entre los años 1982 a 1985 a 21,3 entre $1996-2000^{9}$. La misma tendencia se ha observado en Polonia, en donde Lange et al., analizaron los cultivos en menores de 18 años entre el 1993 a 2002. En este estudio, la prevalencia de OM de pies aumentó de $3 \%$ a $19 \%$, mientras que la de uñas de las manos fue de $0 \%$ a $14 \%{ }^{10}$. Cabe destacar que es difícil determinar si esta tendencia se debe a un aumento en la sensibilidad, al uso de técnicas diagnósticas nuevas o a una mayor sospecha clínica. En Latinoamérica son necesarios estudios similares, centrados en población pediátrica, que permitan confirmar si existe o no este aparente aumento en la prevalencia de OM en niños.

\section{Microbiología}

Las OM son causadas tanto por hongos dermatofitos como no dermatofitos, siendo los primeros los causantes del $90 \%$ de los casos $^{11}$. Los dermatofitos corresponden a un grupo de hongos filamentosos constituido por tres géneros: Epidermophyton, Trichophyton y Microsporum, que destacan porque poseen la capacidad de invadir tejidos ricos en queratina ${ }^{12}$. El 10\% de las OM restantes, son causadas por levaduras e infrecuentemente por mohos (estos últimos pueden infectar a pacientes inmunosuprimidos) ${ }^{11}$. La infección por Candida spp corresponde a una causa común de OM en menores de 3 años y puede ser manifestación de una candidiasis mucocutánea crónica ${ }^{10}$. En OM de manos los agentes etiológicos principales varían entre T. rubrum y Candida albicans, según las series analizadas $^{5,6,10,13}$.

Trichophyton rubrum corresponde al agente aislado con más frecuencia, probablemente debido a que este dermatofito se contagia entre personas. Los dermatofitos pueden permanecer viables por largos períodos de tiempo en piscinas, duchas, toallas, etc., lo que contribuye a su alta contagiosidad.

En Chile, Moreno et al., analizaron 129 cultivos y micológicos directos de niños entre enero 2012 y septiembre 2012, realizados en el Laboratorio de Dermatología del Hospital Clínico de la Universidad de Chile. Del total de muestras de uñas de pie, un $24 \%$ tuvo un cultivo de hongos positivo, siendo el principal agen- 
te aislado T. rubrum, en un $83 \%$ de los $\operatorname{casos}^{7}$. Palma et al., analizaron los cultivos en menores de 15 años, entre el 2006 al 2016, en el mismo laboratorio. En este estudio retrospectivo, de un total de 1.626 cultivos, 417 fueron positivos $(25,6 \%)^{5}$. El principal agente aislado en uñas de pie fue T. rubrum (70\%), seguido por Candida $\operatorname{spp}(22 \%)$, mientras que en manos fue Candida $\operatorname{spp}(66,7 \%)^{5}$. Este es el estudio nacional con el mayor número cultivos para OM realizado hasta la fecha en población infantil. Cabe destacar que los resultados obtenidos en este trabajo son similares a los descritos en la literatura internacional. Lo anterior permitiría replicar esquemas de tratamientos previamente utilizados en otros países ${ }^{10}$.

\section{Clínica}

Un 50\% de las alteraciones ungueales persistentes en niños y adultos corresponden a OM. Dentro de los diagnósticos diferenciales se encuentran la psoriasis ungueal, distrofia ungueal, alopecia areata, dermatitis atópica, liquen plano, trauma, entre otros ${ }^{14-16}$.

Dado que diferenciarlos puede ser complejo, es imprescindible una buena historia clínica, examen físico completo (buscando signos de otras enfermedades dermatológicas) y realizar exámenes microbiológicos para descartar o confirmar la presencia de OM.

Clínicamente, la uña se observa gruesa, amarillenta o blanquecina pudiendo estar desprendida del lecho. Existen diferentes formas de clasificar las $\mathrm{OM}^{11}$, actualmente se clasifican en patrones a partir del punto de inicio de la infección: Distal/lateral subungueal: la infección ingresa por el extremo distal de la uña (figura 1). Blanca superficial: El hongo penetra por la superficie dorsal de la placa ungueal (figura 2). Proximal subungueal: penetración de la infección por el pliegue proximal (se ve en pacientes inmunosuprimidos). Dentro de estos patrones, la presentación más frecuente es la distal/lateral subungueal.

\section{Laboratorio}

Dada la amplia variedad de diagnósticos diferenciales, se recomienda realizar una prueba de confirmación diagnóstica previo al inicio del tratamiento ${ }^{5,15,17,18}$. El método standard consiste en un examen de micológico directo con hidróxido de potasio $(\mathrm{KOH})$, más un cultivo micológico en agar dextrosa Sabouraud ${ }^{18-20}$.

El micológico directo es una prueba operador dependiente, que tiene una variabilidad no solo al momento de interpretar el examen, sino también al momento de tomar la muestra. Posee una sensibilidad global de $60 \%$ y especificidad de $95 \%{ }^{21}$. Su principal ventaja es su bajo costo y la rapidez del resultado. Se realiza desprendiendo material subungueal, al cual se agrega $\mathrm{KOH}$ (queratolítico) y se observa al microscopio de luz. El cultivo requiere de un período de incubación de $2-4$ semanas y su sensibilidad es $56 \%{ }^{21}$, disminuyendo a cifras cercanas a $33 \%$ si el paciente recibió antifúngicos previamente ${ }^{20}$. Su especificidad es $99 \%{ }^{21}$ y es hasta la fecha, el único examen que permite determinar la presencia de agentes causales de diversos tipos: dermatofitos, levaduras y mohos. El micológico directo junto al cultivo hasta la fecha son los métodos clásicos más utilizados y recomendados.

Otro método diagnóstico, disponible en Chile, es la biopsia ungueal, que consiste en obtener muestras de la parte más activa de la infección. Se realiza un corte de la uña y esta se envía a estudio. En el laboratorio la uña se tiñe con hematoxilina eosina y ácido peryódico de Schiff (PAS), buscando observar estructuras fúngicas al microscopio. Posee una sensibilidad cercana

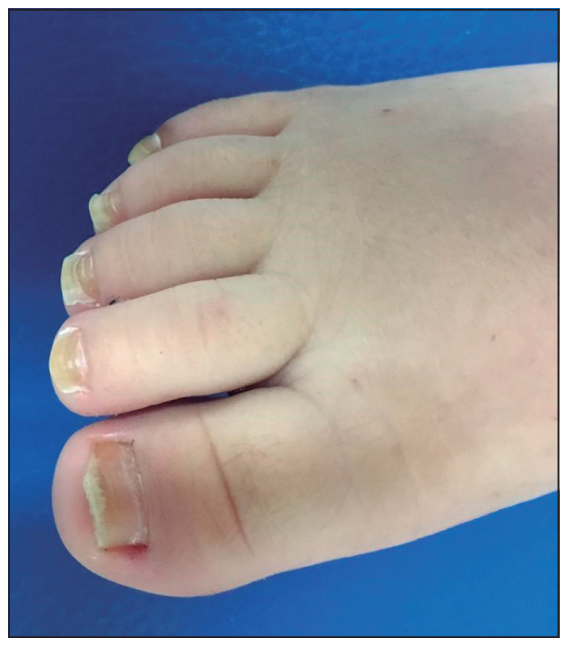

Figura 1. Onicomicosis de pies, en paciente pediátrico, presentación distal/ lateral subungueal.

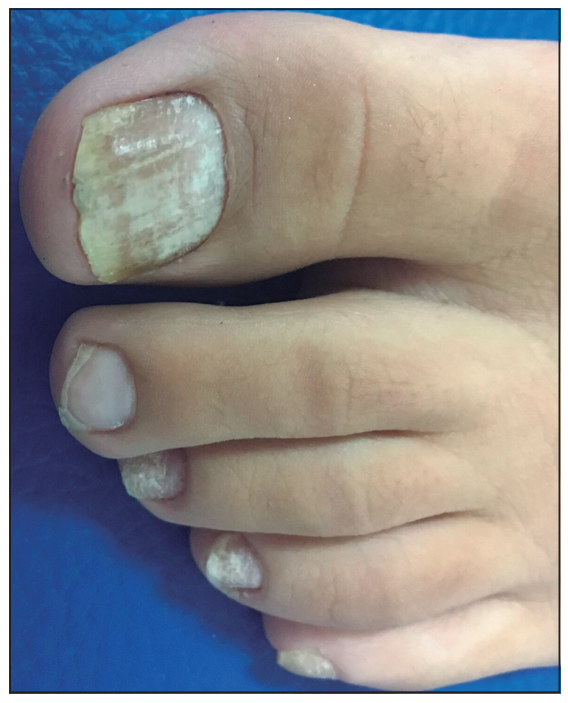

Figura 2. Onicomicosis de pies, en paciente pediátrico, presentación blanca superficial. 
al $84 \%$ y una especificidad de $89 \%{ }^{21}$. Aún cuando en distintas publicaciones ${ }^{19,20}$ y en la revisión sistemática de Velásquez Aguedo et al. ${ }^{21}$ concluyen que la biopsia con PAS es el método más sensible, ésta no identifica el agente etiológico, por lo que se recomienda complementarlo siempre con un cultivo.

El uso de PCR para el diagnóstico de OM se encuentra disponible en Chile de forma preliminar en algunos centros. Su costo es más elevado y los kits disponibles solo identifican dermatofitos. Por lo anterior, su uso de forma rutinaria no se justifica por el momento ${ }^{15-17}$.

El cultivo en Agar para dermatofitos, está dirigido a detectar específicamente la presencia de dermatofitos, pero no identifica el agente específico. No reacciona frente a ciertos tipos de levaduras o mohos ${ }^{22}$ y son varios los agentes contaminantes que pueden dar un falso positivo, por lo que no es el método de elección ${ }^{23}$.

Otra opción es la tinción con blanco de calcoflúor. Esta tinción actúa uniéndose a la celulosa de la pared celular del hongo ${ }^{24}$. La sensibilidad es muy variable según el estudio. Haldane et al., reportaron para dermatofitos una sensibilidad de $92 \%$ y una especificidad de $95 \%$ al analizar 207 muestras $^{25}$. Por otro lado, Bonifaz et al., analizaron 33 muestras, seleccionando pacientes sin otras patologías concomitantes y que no habían recibido tratamiento antifúngico, obteniéndose una sensibilidad de $57 \%{ }^{26}$. La gran desventaja de este método es su costo, dado a que se requiere un microscopio de fluorescencia.

\section{Terapia sistémica}

Los antimicóticos orales se clasifican en familias de acuerdo con su estructura química en: triazoles (itraconazol, fluconazol, voriconazol, etc.), imidazoles (ketoconazol) y alilaminas (terbinafina). Estos actúan interfiriendo con las enzimas encargadas de la síntesis de ergosterol, principal componente de la membrana celular del hongo, pudiendo actuar de forma fungicida o fungistática.

Se recomienda la terapia oral cuando son múltiples las uñas involucradas, hay compromiso de más del $50 \%$ de la placa ungueal o cuando se sospecha que la penetración de medicamentos tópicos va a ser subóptima $^{13}$.

Terbinafina es el antifúngico oral que ha demostrado ser más eficaz en el tratamiento de dermatofitos tricospóricos. Actúa generando un déficit de ergosterol $y$ tiene un efecto fungistático y fungicida ${ }^{27}$. Este antimicótico tiene la capacidad de concentrarse en la uña por períodos prolongados, pudiendo ser detectado en la uña hasta 9 meses después de terminado el tratamiento standard para OM. Actualmente sólo está aprobado por la FDA en mayores de 4 años para el tratamiento de tiña capitis (que en Estados Unidos es predominantemente causada por Tricophyton tonsurans, a diferencia de Chile, donde es causada principalmente por $\mathrm{Mi}$ crosporum canis. Para este último agente, terbinafina no es el tratamiento de elección).

En una revisión sistemática de Gupta et al., el uso de terbinafina en 52 niños menores de 17 años alcanzó tasa de curación, definida como mejoría clínica y microbiológica (cultivo y micológico directo), en un $80,4 \%$ de los $\operatorname{casos}^{28}$. Es importante destacar que la dosis en niños debe ser ajustada por peso (tabla 1). La duración actual recomendada para el tratamiento es 6 semanas para OM de manos y 12 semanas para OM de pies.

Terbinafina es considerada un medicamento seguro tanto en niños, adultos mayores, diabéticos y pacientes VIH positivos. Dentro de los principales efectos adversos reportados en niños se encuentran: urticaria, anorexia, dolor epigástrico, fatiga, erupción vesiculopustular, elevación de transaminasas y agranulocito$\operatorname{sis}^{28-30}$. Estas se normalizan una vez suspendido el tratamiento ${ }^{31}$. Uno de los efectos adversos que causa más preocupación con el uso de terbinafina es el desarrollo de hepatitis, que, si bien es infrecuente, aparece después de 4-6 semanas de iniciado el tratamiento ${ }^{32}$. La función hepática se normaliza una vez suspendido el tratamiento.

Otra opción de tratamiento es el uso de itraconazol. Este actúa inhibiendo la enzima 14- $\alpha$ demetilasa, la cual inhibe la síntesis de ergosterol, lo que da a este agente un efecto fungistático. El itraconazol no induce el citocromo p-450, pero es un potente inhibidor de la enzima CYP3AP, lo que tiene trascendencia clínica a la hora de valorar sus interacciones con otras drogas (tabla 2). Itraconazol tiene un espectro de acción más amplio que terbinafina, siendo más efectivo en tratar Candida albicans ${ }^{33}$. La dosis debe ajustarse por peso (tabla 1). Se puede usar un régimen continuo o en pulsos. El régimen continuo debe durar 6 semanas en OM de manos y 12 semanas en OM de pies. La terapia en pulsos consiste en administrar la dosis correspondiente una vez al día, por 1 semana, descansando 3 semanas hasta la siguiente administración. Se requieren 2 pulsos en OM de manos y 3-4 pulsos para OM en pies (tabla $1)$.

Gupta et al., analizaron la tasa de curación de cada régimen, en población pediátrica, con itraconazol, concluyendo que el régimen continuo tendría una mayor tasa de curación al compararlo con el régimen en pulso $(85,7 \%$ vs $68,4 \%)$. Sin embargo, el tratamiento en pulsos presenta una menor tasa de efectos adver$\operatorname{sos}^{28}$. Dentro de los efectos adversos reportados para itraconazol se encuentran fatiga, gastritis, cefalea y elevación de pruebas hepáticas ${ }^{28-32,34}$. En adultos, efectos adversos más graves como toxicidad hepática e insu- 
Tabla 1. Dosis y esquemas de tratamientos para onicomicosis en niños (14) (39)

\begin{tabular}{|c|c|c|c|}
\hline Antifúngico & Dosis & Duración & Otros \\
\hline \multicolumn{4}{|l|}{ Tópico } \\
\hline Ciclopirox 8\% & Uso diario, retirar 1 vez a la semana & 48 semanas & \\
\hline Amorolfina 5\% & Aplicar 1 o 2 veces a la semana & $24-48$ semanas & Reportes aislados* \\
\hline Eficonazol $10 \%$ & Uso diario & 48 semanas & Sin ensayos* \\
\hline Tavaborol 5\% & Uso diario & 48- 52 semanas & Ensayos aislados* \\
\hline \multicolumn{4}{|l|}{ Oral } \\
\hline \multirow[t]{2}{*}{ Terbinafina } & $<20$ kg: 62.5 mg/día & Manos: 6 semanas & Comprimidos de $250 \mathrm{mg}$ \\
\hline & $\begin{array}{l}\text { 20-40 kg: } 125 \text { mg/día } \\
>40 \text { kg: } 250 \text { mg/día }\end{array}$ & Pies: 12 semanas & \\
\hline \multirow[t]{4}{*}{ Itraconazol } & Pulso: 5 mg/kg/día & Manos: 2 pulsos & Cápsulas de 100 mg \\
\hline & & Pies: 3 pulsos & \\
\hline & Continuo: 5 mg/kg/día & Manos: 6 semanas & Pulso: dosis por 1 semana, luego descanso \\
\hline & Dosis máxima: 400 mg/día & Pies: 12 semanas & de 3 semanas \\
\hline
\end{tabular}

*Realizados en población pediátrica.

Tabla 2. Interacción medicamentosa de itraconazol (Sporanox) (35,42 $^{32}$

\begin{tabular}{|c|c|}
\hline Clase & Ejemplos de medicamentos* \\
\hline Alfa-bloqueadores & Tamsulosina \\
\hline Ansiolíticos & Buspirona \\
\hline Antiarrítmicos & Digoxina, dofetilida, quinidina, disopiramida \\
\hline Antibióticos & Claritromicina, eritromicina \\
\hline Anticoagulantes & Warfarina, apixabán, rivaroxabán, dabigatrán, cilostazol \\
\hline Anticonceptivos & Dienogest \\
\hline Anticonvulsivantes & Carbamazepina, fenobarbital, fenitoína \\
\hline Antidepresivos & Venlafaxina \\
\hline Antidiarreicos & Loperamida \\
\hline Antinflamatorios & Meloxicam \\
\hline Antimicobacterias & Rifabutina, isoniacida, rifampicina \\
\hline Antineoplásicos & Busulfán, docetaxel, alcaloides de la vinca \\
\hline Antipsicóticos & Pimozida, quietapina, risperidona \\
\hline Benzodiazepinas & Alprazolam, diazepam, midazolam, triazolam, \\
\hline Beta-2-agosnista & Salmeterol \\
\hline Beta-bloqueadores & Nadolol \\
\hline Bloqueadores de canales de calcio & Dihidropiridínicos, verapamilo \\
\hline Corticoesteroides & Metilprednisolona, budesónida (inhalada y vía oral), dexametasona, fluticasona (inhalada y nasal) \\
\hline Ergotamínicos & No especificado \\
\hline Estatinas & Atorvastatina, cerivastatina, lovastatina, simvastatina \\
\hline Hipnóticos & Zopiclona \\
\hline Hipoglicemiantes orales & Repaglinida, saxagliptina \\
\hline Inhibidores de proteasas & Indinavir, ritonavir, saquinavir \\
\hline $\begin{array}{l}\text { Inhibidores de transcriptasa inversa } \\
\text { no nucleósidos }\end{array}$ & Nevirapina \\
\hline Inmunosupresores & Ciclosporina, tacrolimus, sirolimus \\
\hline Opioides & Fentanilo, alfentanilo, levacetilmetadol \\
\hline Procinéticos & Cisaprida \\
\hline Retinoides & Alitretinoína \\
\hline Supresores del ácido gástrico & Antagonistas del receptor $\mathrm{H} 2$, inhibidores de la bomba de protones \\
\hline Otros & Trimetrexato, halofantrina, cilostazol, anfotericina B, eletriptán, ivabradina, sindenafilo \\
\hline
\end{tabular}

*Reportados por Sporanox, no completamente inclusivo. 
ficiencia cardíaca han sido reportados ${ }^{35}$. Son múltiples las interacciones medicamentosas que presenta este medicamento, por lo que se debe decidir su uso con precaución ${ }^{35}$ (tabla 2).

Fluconazol actúa de manera similar a itraconazol, pero dentro de sus ventajas se encuentra que presenta un escaso metabolismo hepático y que se administra semanalmente. En el tratamiento de OM tiene menor eficacia al compararlo con terbinafina e itraconazol, logrando una curación cercana a $45 \%{ }^{28}$. No se considera terapia de primera línea para OM por Candida spp, dado que la concentración residual en las uñas es escasa, requiriendo períodos prolongados de tratamiento: de 6 a 9 meses en $\mathrm{OM}$ de manos y 9-18 meses en $\mathrm{OM}$ de pies $^{33}$.

La alteración de las pruebas hepáticas con el uso de antimicóticos como terbinafina ${ }^{32}$, itraconazol ${ }^{34,36}$ y fluconazol ${ }^{37}$, es un efecto adverso descrito tanto en niños como en adultos, lo que refuerza la necesidad de solicitar un control de exámenes antes y durante el tratamiento. Además, es importante advertir sobre la prohibición del consumo de bebidas alcohólicas, especialmente en adolescentes ${ }^{29,30}$.

Si bien hasta la fecha no existen guías clínicas para el manejo de $\mathrm{OM}$ en niños, se recomienda que antes del inicio del tratamiento se realice un control de pruebas hepáticas y que este se repita a las 4 a 6 semanas del tratamiento.

\section{Terapia tópica}

Actualmente, en Chile existen 2 opciones disponibles en formato laca, aprobadas por la FDA, para el tratamiento de la OM: laca de ciclopirox $8 \%$ y laca amorolfina $5 \%$.

Ciclopirox es un tipo de hidroxipiridona, que interfiere en diversas vías metabólicas de la célula fúngica. Esta laca se debe aplicar sobre la uña diariamente en la noche, siendo removido una vez a la semana con alcohol, momento en el que también se debe recortar la uña ${ }^{13}$. La duración del tratamiento es de 3 meses $^{29}$. Si bien en adultos la eficacia es modesta, en niños la respuesta es considerable.

Friedlander et al., realizaron un ensayo clínico randomizado, doble ciego, controlado con placebo, en 40 niños, observándose un $71 \%$ de efectividad con ciclopirox a las 32 semanas de utilización en población pediátrica. Como efecto adverso se reportó una coloración amarilla cafesosa de la placa ungueal en un paciente ${ }^{38}$.

La amorolfina actúa inhibiendo enzimas que participan en la síntesis de ergosterol, lo que lleva a la inhibición del crecimiento y muerte celular. Para utilizarla, la uña se debe limar antes de aplicar el producto, lim- piarse, tras lo cual la laca se aplica una vez a la semana, por 6 a 12 meses. El uso de amorolfina solo ha sido reportado en dos casos pediátricos ${ }^{30}$.

Nuevos antifúngicos tópicos han sido aprobados por la FDA: efinaconazol y tavaborol, no disponibles en Chile. En niños, hay un estudio etapa IV para tavaborol $^{39}$, donde se evaluó tavaborol $5 \%$, luego de usarlo diariamente por 48 semanas, en 47 niños de 6 a 16 años. Un 8,5\% de los pacientes tuvo una cura completa, con micológico directo y cultivo negativos, además de mejoría clínica. Un 14,9\% alcanzó una cura casi completa, con exámenes negativos, pero rastro de decoloración ungueal.

\section{Prevención de recurrencias}

La recurrencia de $\mathrm{OM}$ en adultos se ha estimado entre un $10 \%$ a $53 \%{ }^{40}$. En niños este porcentaje se desconoce. Sin embargo, existen factores que predisponen a la recurrencia. Dentro de estos se encuentran: inmunosupresión, presencia de tiña pedis, historia familiar de OM, humedad, calzado oclusivo, higiene deficiente, trauma ungueal, tratamiento incorrecto de la infección original y baja adherencia al tratamiento ${ }^{41}$.

Para evitar las recurrencias se recomienda mantener los pies limpios y secos, uñas cortas, desechar calzado antiguo, utilizar calcetines de material absorbente, no caminar descalzo en lugares húmedos como duchas públicas y piscinas. Se debe lavar el calzado con agua $>60^{\circ} \mathrm{C}$ por más de 45 minutos, opción disponible al utilizar máquinas lavadoras ${ }^{40}$. Mención especial merece el tratamiento de familiares con $\mathrm{OM}$, dado a que serían la fuente de reinfección en un $86 \%$ de los $\operatorname{casos}^{29,41}$.

\section{Conclusiones}

La OM en niños es una patología cuya prevalencia va en aumento y debe ser parte del diagnóstico diferencial al observar alteraciones ungueales en niños. Su diagnóstico se basa en la sospecha clínica asociado a la positividad de exámenes microbiológicos, clásicamente micológico directo y cultivo micológico. Respecto del tratamiento en niños, una alternativa con pocos efectos adversos es el uso de tratamientos tópicos con lacas por períodos prolongados (ciclopirox $8 \%$ o amorolfina $5 \%$ ). En el caso de requerirse terapia oral, es esencial tener la confirmación microbiológica para iniciar la terapia. Terbinafina es medicamento de elección en tricofitos, sin embargo, al utilizarla se debe considerar la monitorización con pruebas hepáticas y hemograma al inicio y a las 4-6 semanas de tratamiento. La segunda opción de tratamiento es itraconazol, 
pero éste presenta un perfil de seguridad menor, ya que presenta más interacciones medicamentosas. Fluconazol es menos efectivo y dada su baja concentración en las uñas debe utilizarse por períodos prolongados, pero sería una alternativa en pacientes con daño hepático previo que requieran tratamiento oral.

Finalmente, algo fundamental a realizar por el pe- diatra, es la educación sobre medidas preventivas y el tratamiento de aquellos familiares que podrían ser la fuente de la infección.

\section{Conflicto de intereses}

Los autores declaran no tener conflicto de intereses.

\section{Referencias}

1. Gupta AK, Daigle D, Foley KA. The prevalence of culture-confirmed toenail onychomycosis in at-risk patient populations. J Eur Acad Dermatol Venereol. 2015;29:1039-44.

2. Gupta AK, Sibbald RG, Lynde CW, et al. Onychomycosis in children: Prevalence and treatment strategies. J Am Acad Dermatol. 1997;36:395-402.

3. Rodríguez-Pazos L, Pereiro-Ferreirós MM, Pereiro M Jr, Toribio J. Onychomycosis observed in children over a 20-year period. Mycoses. 2011;54:450-3.

4. Totri CR, Feldstein S, Admani S, Friedlander SF, Eichenfield LF. Epidemiologic analysis of onychomycosis in the San Diego pediatric population. Pediatr Dermatol. 2017;34:46-9.

5. Palma C, Zemelman V, Stevens J, Araníbar L, Zapata S, Hernández A. Análisis epidemiológico de onicomicosis diagnosticada por laboratorio en pacientes menores de 15 años. Rev Hosp Clín Univ Chile. 2017;28:177-80.

6. Wlodek C, Trickey A, de Berker D, Johnson E. Trends in pediatric laboratorydiagnosed onychomycosis between 2006 and 2014 in the southwest of England. Pediatr Dermatol. 2016;33:358-9.

7. Moreno C, Zapata S, De la Parra R, Tapia C. Estudio micológico de muestras de uñas en población pediátrica y adolescente. Experiencia de un año en el Laboratorio de Dermatología, Hospital Clínico Universidad de Chile. Rev Chil Dermatol. 2014;30:271-4.

8. Vásquez-Del Mercado E, Arenas R. Onicomicosis en niños. Estudio retrospectivo de 233 casos mexicanos. Gac Med Mex. 2008;144:7-10.

9. Sigurgeirsson B, Kristinsson KG, Jonasson PS. Onychomycosis in Icelandic children. J Eur Acad Dermatol Venereol. 2006;20:796-9.

10. Lange M, Roszkiewicz J, SzczerkowskaDobosz A, Jasiel-Walikowska E, Bykowska $B$. Onychomycosis is no longer a rare finding in children. Mycoses. 2006;49:559.

11. Scher R, Rich P, Elewski B, Pariser D. The epidemiology, etiology, and pathophysiology of onychomycosis. Semin Cutan Med Surg. 2013;32:2-4.

12. Del Palacio A, Garau M, Cuétara MS. Tratamiento actual de las dermatofitosis. Rev Iberoam Micol. 2002;19:68-71.

13. Singal A, Khanna D. Onychomycosis: Diagnosis and management. Indian J Dermatol Venereol Leprol. 2011;77:65972.

14. Gupta AK, Skinner AR. Onychomycosis in children: A brief overview with treatment strategies. Pediatr Dermatol. 2004;21:74-9.

15. Solís-Arias MP, García-Romero MT. Onychomycosis in children. A review. Int J Dermatol. 2017;56:123-30.

16. Soto R. ¿Por qué fallan los tratamientos para onicomicosis? Rev chil dermatol. 2011;27:140-5

17. Gatica JL, Muñoz L, Zapata S, Sazunic I. Onicomicosis: Examen histológico una herramienta diagnóstica. Rev Chil Dermatol. 2011;27:242-6.

18. Fuller LC, Barton RC, Mohd Mustapa MF, Proudfoot LE, Punjabi SP, Higgins EM. British Association of Dermatologists guidelines for the management of tinea capitis 2014. Br J Dermatol. 2014;171:45463.

19. Haghani I, Shokohi T, Hajheidari Z, Khalilian A, Aghili SR. Comparison of diagnostic methods in the evaluation of onychomycosis. Mycopathologia. 2013;175:315-21.

20. Wilsmann-Theis D, Sareika F, Bieber $\mathrm{T}$, Schmid-Wendtner MH, Wenzel J. New reasons for histopathological nailclipping examination in the diagnosis of onychomycosis. J Eur Acad Dermatol Venereol. 2011; 25:235-7.

21. Velasquez-Agudelo V, Cardona-Arias JA. Meta-analysis of the utility of culture, biopsy, and direct $\mathrm{KOH}$ examination for the diagnosis of onychomycosis. BMC Infect Dis. 2017;17:166.

22. Elewski BE, Leyden J, Rinaldi MG, Atillasoy E. Office practice-based confirmation of onychomycosis: A US nationwide prospective survey. Arch Intern Med. 2002;162:2133-8.

23. Daniel CR 3rd, Elewski BE. The diagnosis of nail fungus infection revisited. Arch Dermatol. 2000;136:1162-4.

24. Yadav S, Saxena AK, Capoor MR,
Ramesh V. Comparison of direct microscopic methods using potassium hydroxide, periodic acid Schiff, and calcofluor white with culture in the diagnosis of onychomycosis. Indian J Dermatol Venereol Leprol. 2013;79:2423.

25. Haldane DJ, Robart E. A comparison of calcofluor white, potassium hydroxide, and culture for the laboratory diagnosis of superficial fungal infection. Diagn Microbiol Infect Dis. 1990;13:337-9.

26. Bonifaz A, Ríos-Yuil JM, Arenas R, et al. Comparison of direct microscopy, culture and calcofluor white for the diagnosis of onychomycosis. Rev Iberoam Micol. 2013;30:109-11.

27. Van Duyn Graham L, Elewski BE. Recent updates in oral terbinafine: Its use in onychomycosis and tinea capitis in the US. Mycoses. 2011;54:679-85.

28. Gupta AK, Paquet M. Systemic antifungals to treat onychomycosis in children: a systematic review. Pediatr Dermatol. 2013;30:294-302.

29. Gupta AK, Mays RR, Versteeg SG, Shear $\mathrm{NH}$, Friedlander SF. Onychomycosis in children: Safety and efficacy of antifungal agents. Pediatr Dermatol. 2018;35:552-9.

30. Feldstein S, Totri C, Friedlander SF. Antifungal therapy for onychomycosis in children. Clin Dermatol. 2015;33:333-9.

31. Aguilar C, Mueller KK. Reversible agranulocytosis associated with oral terbinafine in a pediatric patient. J Am Acad Dermatol. 2001;45:632-4.

32. Patel D, Castelo-Soccio LA, Rubin AI, Streicher JL. Laboratory monitoring during systemic terbinafine therapy for pediatric onychomycosis. JAMA Dermatol. 2017;153:1326-7.

33. Gupta AK, Paquet M, Simpson FC. Therapies for the treatment of onychomycosis. Clin Dermatol. 2013;31:544-54.

34. Gupta AK, Nolting S, de Prost $Y$, et al. The use of itraconazole to treat cutaneous fungal infections in children. Dermatology. 1999;199:248-52.

35. Sporanox (itraconazole) capsules [Internet]. [Citado el 16 de febrero de 2019] p.1-33. Disponible en:http://www. accessdata.fda.gov/drugsatfda_docs/label/ 2012/020083s048s049s050lbl.pdf. 
36. Heikkilä H, Stubb S. Onychomycosis in children: treatment results of fortyseven patients. Acta Derm Venereol. 2002;82:484-5.

37. Egunsola O, Adefurin A, Fakis A, JacqzAigrain E, Choonara I, Sammons H. Safety of fluconazole in paediatrics: a systematic review. Eur J Clin Pharmacol. 2013;69:1211-21

38. Friedlander SF, Chan YC, Chan YH, Eichenfield LF. Onychomycosis does not always require systemic treatment for cure: a trial using topical therapy. Pediatr Dermatol. 2013;30:316-22.

39. Rich P, Spellman M, Purohit V, Zang C, Crook TJ. Tavaborole 5\% Topical solution for the treatment of toenail onychomycosis in pediatric patients: results from a phase 4 open-label study. J Drugs Dermatol. 2019;18:190-5.

40. Piraccini BM, Sisti A, Tosti A. Long-term follow-up of toenail onychomycosis caused by dermatophytes after successful treatment with systemic antifungal agents. J Am Dermatol. 2010;62:411-4.

41. Gupta AK, Cernea M, Foley KA. Improving cure rates in onychomycosis. J Cutan Med Surg. 2016;20:517-31.

42. Sporanox (itraconazole) capsules [Internet]. [Citado el 13 de junio de 2019] p. 13-9. Disponible en:https://www. accessdata.fda.gov/drugsatfda_docs/label/ 2017/020083s061,020657s036lbl.pdf. 